Research Article

\title{
Deep Learning-Based MRI in Diagnosis of Fracture of Tibial Plateau Combined with Meniscus Injury
}

\author{
Xiaoxiao Xie $\mathbb{D}$, ${ }^{1}$ Zhen Li $\mathbb{D},{ }^{1}$ Lu Bai $\mathbb{D},{ }^{1}$ Ri Zhou $\mathbb{D},{ }^{1}$ Canfeng Li $\mathbb{D},{ }^{1}$ Xiaocheng Jiang $\mathbb{D},{ }^{1}$ \\ Jianwei Zuo $\left(\mathbb{C}^{1},{ }^{1}\right.$ and Yulong Qi $\mathbb{( i )}^{2}$ \\ ${ }^{1}$ Department of Sports Medicine, Peking University Shenzhen Hospital, Shenzhen 518036, Guangdong, China \\ ${ }^{2}$ Department of Medical Imaging, Peking University Shenzhen Hospital, Shenzhen 518036, Guangdong, China \\ Correspondence should be addressed to Yulong Qi; xiexiaoxiao@xiexiaoxiao724.com
}

Received 27 September 2021; Revised 25 November 2021; Accepted 27 November 2021; Published 20 December 2021

Academic Editor: M Pallikonda Rajasekaran

Copyright (C) 2021 Xiaoxiao Xie et al. This is an open access article distributed under the Creative Commons Attribution License, which permits unrestricted use, distribution, and reproduction in any medium, provided the original work is properly cited.

This study aimed to explore the application value of magnetic resonance imaging (MRI) images based on deep learning algorithms in the diagnosis of tibial plateau fractures combined with meniscus injuries. The original MRI image was input into the deep learning convolutional neural network (CNN), and the knee joint undersampled and fully sampled MRI image data were used for training to obtain a neural network model that can effectively remove the noise and blur of the undersampled image. Then, the image was reconstructed by the Regridding model to obtain an image with less noise and clearer structure. At the same time, all subjects underwent knee MRI examinations, and algorithms were used to analyze the sensitivity, specificity, and accuracy of their images. It was found that of 160 menisci from 80 cases of tibial plateau fractures, 64 were normal meniscus and 88 were injured menisci. The sensitivity, specificity, and accuracy of optimized MRI in diagnosing fracture of tibial plateau combined with meniscus injury were $96.9 \%, 93.2 \%$, and $95.3 \%$, respectively. In conclusion, the restored MRI images have high sensitivity in the diagnosis of meniscus injury and high consistency with the intraoperative results. It suggests that the optimized MRI image is effective in the diagnosis of meniscus injury.

\section{Introduction}

The knee joint is a typical trochlear joint. It is the largest and most complex joint that bears the weight of the body. It is composed of the lower end of the femur, the upper end of the tibia, the patella, and the meniscus $[1,2]$. As the weightbearing area between the femur and the tibia, the meniscus has the functions of cushioning, lubricating, absorbing energy, and reducing the pressure between the tibia and the femur in daily life. Fracture of tibial plateau is a kind of intraarticular knee fracture caused by indirect or direct violence. When violence directly hits the inner or outer side of the knee joint, the knee joint valgus or varus will occur, leading to the tibial plateau fractures. The meniscus is extremely vulnerable to injury due to the special position in the knee joint [3]. Meniscus injuries are more common in knee joint injuries. Generally, meniscus injuries can be caused by chronic degeneration or severe trauma and can also be complicated by other soft tissue injuries of the knee, such as joint capsule injury and cartilage injury. Usually, this is also the cause of swelling after partial injury of the knee joint. When the knee joint is flexed, the medial meniscus moves backward, and when the knee joint is stretched, the meniscus moves forward. When this contradictory movement goes beyond the normal range, meniscus injury and tear may occur.

The diagnosis and treatment of this kind of bone structure combined with soft tissue damage are complicated. At this time, improper treatment or failure to find the meniscus damage in time will cause traumatic osteoarthritis in the follow-up, which will further cause severe joint dysfunction, affecting the progress of the patient's treatment and postoperative rehabilitation. Worse still, it will cause unnecessary economic losses and psychological pressure. According to related studies, tibial plateau fractures combined with meniscus injuries have become increasingly 
common, with the incidence ranging from $47 \%$ to $99 \%$ [4]. Therefore, the correct diagnosis of meniscus and other soft tissue injuries before surgery is of great significance. Nowadays, clinical diagnosis methods for meniscus and other soft tissue injuries include arthroscopy, MRI, and knee B-ultrasound $[5,6]$. Although arthroscopy is very accurate in diagnosing this type of soft tissue injury, this method is a traumatic and will cause more pain and wound complications. The accuracy of the knee joint B-ultrasound diagnosis is not high enough, and thus its clinical use is also limited. Magnetic resonance imaging (MRI) has high resolution for the soft tissue of the knee joint [7] and has high specificity and sensitivity for the diagnosis of injury sites. More importantly, MRI examination is noninvasive with low radiation $[8,9]$, so MRI is widely used to diagnose meniscus injuries in clinical practice.

Nevertheless, the MRI diagnosis results are often different from the real situation clinically. It may be because during MRI examination, the image will appear blurred and has artifacts due to the movement of limbs, the special examination site, heartbeat, and breathing. CNN is a typical representative of feedforward neural network, and it performs particularly well in image processing including image classification, target detection, and image segmentation. Above, in this study, a convolutional neural network (CNN)-based algorithm was proposed to restore the image. The image restoration model based on the Regridding method can retain rich image features. Hence, it was used to improve the quality of MRI images of meniscal injury to analyze the application value of MRI image based on deep learning in diagnosing meniscus injury and expected to provide theoretical basis and data for follow-up related clinical operations.

\section{Materials and Methods}

2.1. Research Objects. 80 patients diagnosed with tibial plateau fractures were selected as research subjects, of whom 48 were male patients and 32 were female patients, aged between 24 and 67 years. The tibial plateau fractures in 44 patients arose from traffic trauma, and the remaining 36 patients fell to have tibial plateau fractures. According to the 6-classification method proposed by Schatzker in 1974 based on fracture X-rays, the subjects were divided into 6 types: 8 cases of type I, 12 cases of type II, 16 cases of type III, 4 cases of IV, 8 cases of type V, and 32 cases of type VI. None of the above patients who participated in this study had undergone knee surgery and had undergone MRI and intraoperative meniscus examination during the treatment.

Inclusion criteria are as follows: (I) patients with tibial plateau fractures combined with meniscus injury; (II) aged 16-70 years; (III) lateral plateau fractures showed significant displacement or collapse, and the medial plateau articular surface remained relatively intact; (IV) patients not having the over-knee surgery; and (V) patients without surgical contraindications.

Exclusion criteria are as follows: (I) patients with other knee joint diseases and severe knee joint deformities; (II) patients with coagulation dysfunction or those unable to have the surgery for other reasons; and (III) patients who did not sign the surgical consent form.

The experiment had been approved by the ethics committee of hospital. The participants and their families had been informed of the experiment related matters, and they had all signed informed consent.

\subsection{MRI Examination and Diagnostic Criteria of Knee Joint.} The magnetic resonance scanning system was used. The patient lied supine on the examination bed, with the knee joint kept straight, and the calf was fixed with a sandbag. The knee joint was externally rotated by $20^{\circ}-30^{\circ}$. The patient should keep still during the scan.

As per the Schatzker classification [10], Schatzker type I fractures are lateral platform split fractures, have no articular surface collapse, and mostly occur in young patients without osteoporosis; Schatzker type II fractures are lateral platform split collapse, usually occurring in patients around 40 years of age or older; Schatzker type III fracture is simple lateral platform collapse, it may occur in any part of the articular surface, but it is often the central area; Schatzker type IV fracture is generally caused by high-energy violence, such as knee dislocation and lateral meniscus injury, and it is relatively rare in clinical practice; Schatzker $\mathrm{V}$ fractures are bicondylar fracture, with varying degrees of articular surface collapse and displacement; Schatzker Type VI fractures are mostly caused by high-energy violence, generally bicondylar fractures combined with metaphyseal fractures, which are more common in high falls and are prone to osteofascial compartment syndrome and vascular and nerve injury.

2.3. Regridding Model. MRI technology collects data in the spatial frequency domain and reconstructs the image. Because the data collected in the spatial frequency domain are not distributed at an equal interval, the image cannot be quickly reconstructed through the Fourier transform. Therefore, the collected data need to be gridded before. The Jackson gridding algorithm is widely recognized [11] because of its simple calculation and less computing time, expressed as follows:

$$
\begin{aligned}
& F_{n}(\varphi, v)=\{[F(\varphi, v) k \cdot \operatorname{Tn}(\varphi, v)] * C(\varphi, v)\} \cdot n(\varphi, v), \\
& T_{n}(\varphi, v)=\frac{T(\varphi, v)}{T(\varphi, v * t C n \varphi, v)}
\end{aligned}
$$

where $F_{n}$ represents the resampled magnetic resonance image data, $F$ represents the MRI data sampled at different intervals, $T_{n}$ represents the density compensation function of uneven sampling, $C$ represents the convolution function, $n$ represents the sampling function at equal intervals, and $T$ represents the sampling at unequal intervals. - stands for multiplication, and * stands for convolution operation.

The function used in the Jackson gridding algorithm is the Kaiser-Bessel function, expressed as follows: 


$$
\begin{aligned}
& s(\varphi)=\frac{\operatorname{do}(\alpha \sqrt{1-(2 \phi / E)} \wedge 2)}{d_{0}(\alpha)},|\varphi| \leq \frac{E}{2}, \\
& s(\varphi)=0,|\varphi| \leq \frac{E}{2},
\end{aligned}
$$

where $s(\varphi)$ represents the Kaiser-Bessel function, do represents the zero-order deformed Bessel function, and $E$ represents the window length.

The MRI image after reconstruction by the grid algorithm is expressed as follows:

$$
h_{m}(x, y)=h_{n}(x, y) \cdot \frac{p(x, y)}{c(x, y)},
$$

where $h_{m}$ is the image reconstructed by the algorithm, $h_{n}$ represents the Fourier transform of $F_{n}, c$ represents the inverse Fourier transform, and $p$ represents the rectangular function.

With the further research and innovation of the gridding algorithm, the gridding algorithm has been improved, and the optimized gridding algorithm is named Regridding algorithm [12]. It is then applied to reconstruct MRI images, expressed as follows:

$$
M r=\varphi,
$$

where $M$ represents the sine interpolation coefficient matrix, $r$ represents the $n$ vector on the spatial data points on the grid, and $\phi$ represents the $m$ vector on the spatial data points on any trajectory. Because the calculation amount is too large, the approximation is iteratively solved.

$$
\widehat{r}=M * D \varphi .
$$

Among them, $\widehat{r}$ represents the approximate solution of $r$, * is the Hermitian transpose, $D=\operatorname{diag}(d)$ represents the diagonal matrix, and $d$ is the density-weighted m-dimensional vector. Then, the following equation is obtained by combining equations (4) and (5).

$$
\widehat{r}=M * \mathrm{DMr} \text {. }
$$

When the value of $M * D$ is infinitely close to $M, d$ is the optimal solution at this moment, expressed as follows:

$$
d=\operatorname{diag}\left(\theta\left[\begin{array}{cc}
f_{k} & 0 \\
0 & 0
\end{array}\right] \theta^{*}\right) .
$$

The matrix $M$ is expressed as follows:

$$
M=\text { TRUR } *,
$$

where Trepresents the Kaiser-Bessel interpolation matrix, $R$ represents the Fourier matrix, $U$ represents the diagonal matrix, and RUR * represents the deconvolution result. The following equation is then obtained by combining equations (4) and (8).

$$
\begin{aligned}
\operatorname{TRURr} & =* \varphi, \\
\widehat{r} & =\operatorname{TRU} * R * D \varphi .
\end{aligned}
$$

Because the value of do will be affected by the interpolation function, after further improvement, the iterative calculation method is used to optimize, and the initialization estimation of do is improved. Starting from $d=$ do, an accurate estimate value is obtained.

$$
d=\frac{d}{H H} * d .
$$

A $H H * d$ value closer to 1 indicates a better density weighting result.

The undersampled MRI image is reconstructed by the final Regridding image reconstruction algorithm.

$$
\mathrm{MRH}=F(\mathrm{MRL}) .
$$

where MRL represents the reconstructed MRI image of undersampled spatial data, which contains a lot of noise and blur, MRH represents the reconstructed fully sampled MRI image, and $F$ represents the function learned by CNN.

A CNN model is used to process undersampled images, and finally fully sampled MRI images are obtained. The calculation equation and structure are shown in Figure 1.where $y_{i}$ represents the undersampled MRI image, $T_{1}, T_{2}$, and $T_{3}$ represent the weight, $a_{1}, a_{2}$, and $a_{3}$ represent the offset, $*$ represents the convolution operation, ReLU represents the activation function [13], and $B M$ represents the regularization operation [14].

$$
\begin{aligned}
y_{0}= & y_{i}+T_{3} * B M\left(\operatorname { R e L U } \left(T_{2} * B M\left(\operatorname{ReLU}\left(T_{1} * y_{i}+a_{1}\right)\right)\right.\right. \\
& \left.\left.+a_{2}\right)\right)+a_{3} .
\end{aligned}
$$

The loss function of the network model is expressed as follows:

$$
f(Y, Z)=\frac{1}{N} * \sum_{j=1}^{N}+\left|Y_{j}-Z_{j}\right|,
$$

where $Y_{j}$ is the pixel in the undersampled image, $Z_{j}$ represents the pixel in the fully sampled image, $N$ represents the number of pixels in the MRI image, and $f(Y, Z)$ represents the loss function.

2.4. Statistical Processing. SPSS19.0 software was used for statistical analysis. Measurement data conforming to the normal distribution were represented by the mean\pm standard deviation, and the difference between groups was analyzed by independent sample $t$-test. Measurement data not conforming to the normal distribution were expressed by the median value and four-point position, and nonparametric rank sum test was used to analyze the difference between groups. Count data were expressed by $n(\%)$, and the differences between groups adopted the chi-square test. $P<0.05$ was the threshold for significance. 


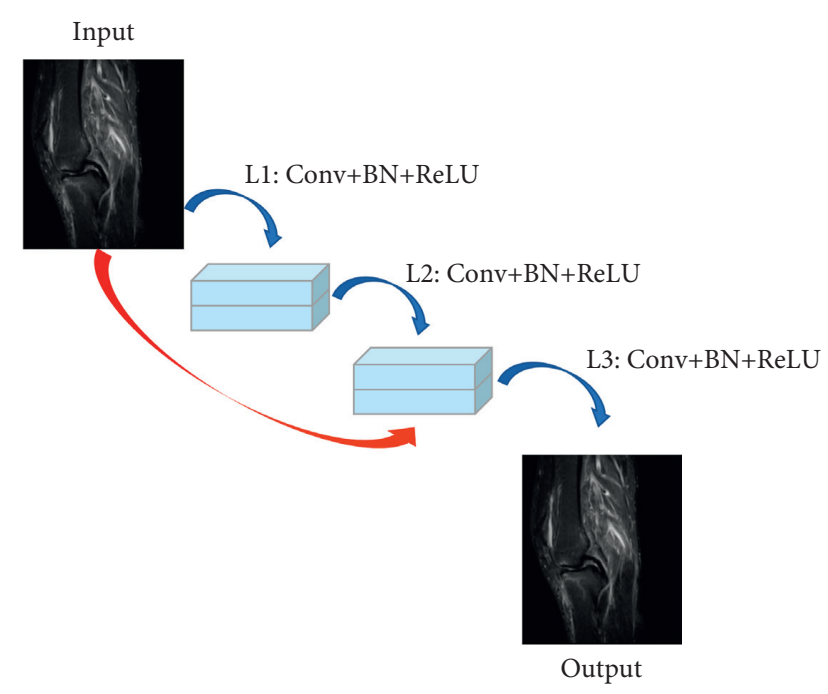

Figure 1: Schematic diagram of the CNN structure.

\section{Results}

3.1. Reconstructed and Optimized Images Based on Deep Learning. The Regridding model image reconstruction algorithm was used to optimize the knee joint MRI undersampled images, and a fully sampled MRI image was obtained. It was found that the undersampled MRI image contained obvious noise and blur, and some structural images were blurry. After the image was reconstructed by the Regridding model, an MRI image with less noise and clearer structure was obtained, as shown in Figure 2.

3.2. CNN Model. The undersampled images were processed by the CNN model, and the knee joint undersampled and fully sampled MRI image data were used for training to obtain a neural network model that can effectively remove the noise and blur of the undersampled image. The MRI images processed by the optimized neural network model are shown in Figures 3 and 4. Figure 3 shows that the fuzzy part was restored by $\mathrm{CNN}$, and Figure 4 shows that the shadowed part was restored by $\mathrm{CNN}$.

\subsection{The Diagnostic Efficacy of Reconstructed MRI Images for} Meniscus Injury. Of the 160 meniscuses of 80 patients with tibial plateau fracture combined with meniscus injury, there were 64 normal meniscuses and 88 tibial plateau fracture combined with meniscus injury after analysis and diagnosis, 4 were diagnosed as tibial plateau fracture combined with meniscus injury by MRI images but were not confirmed during surgery, and 2 were found to be tibial plateau fracture combined with meniscus injury but were not diagnosed during MRI images analysis, as shown in Figure 5. Taking the diagnosis result during surgery as the gold standard, the sensitivity, specificity, and accuracy of optimized MRI in diagnosing tibial plateau fracture combined with meniscus injury were $96.3 \%, 93.4 \%$, and $96.1 \%$, respectively. Hence, the optimized MRI images had high sensitivity and accuracy.
The consistency between MRI images and intraoperative condition was compared. The results are shown in Figure 6.

3.4. Meniscus Tear Classification Based on the Reconstructed MRI Image. For the 160 menisci from 80 cases of tibial plateau fracture combined with meniscus injury, the type of meniscus tear was diagnosed, and the MRI classification results were compared with intraoperative observation results to analyze the consistency between the two, as shown in Figure 7. The consistency of horizontal tear was 0.63 , transverse tear was 0.64 , longitudinal tear was 0.78 , oblique tear was 0.96 , and undetected tear was 0.99 .

\section{Discussion}

The meniscus is a half-moon-shaped bone located on the inner and outer sides of the tibial joint. The edge part is thicker, and the center part is thinner. The edge part of the meniscus can be repaired slowly after being damaged, but it cannot be repaired by itself after severe rupture. However, after the severely damaged meniscus is removed, a fibrocartilage meniscus can be regenerated through the synovium [15]. The outer third of the normal meniscus has many blood vessels, called the red zone, and the middle third has a few capillaries, called the red-white zone, and there are almost no capillaries on the inner side, called the white area [16]. When using MRI to scan the meniscus, each sequence of MRI shows a low signal [17]. When scanning the meniscus with a thickness of $4 \mathrm{~mm}$, the meniscus exhibits a uniform low signal. Tibial plateau fracture combined with meniscus injury is common in knee joint injuries. Tibial plateau fracture combined with meniscus injury can be caused by chronic degeneration or severe trauma and can also be complicated by other soft tissue injuries of the knee, such as joint capsule injury and cartilage injury. Usually, this is also the cause of swelling after partial injury of the knee joint. When the knee joint is flexed, the medial meniscus moves backward, and when the knee joint is stretched, the meniscus moves forward. When this contradictory movement goes beyond the normal range, tibial plateau fracture combined with meniscus injury and tear may occur. Related research shows that the meniscus is extremely vulnerable to injury due to the special position in the knee joint $[18,19]$. In order to improve the diagnostic efficiency of tibial plateau fractures, deep learning was introduced to process MRI images of tibial plateau fractures.

MRI is a widely used method to diagnose tibial plateau fracture combined with meniscus injury with high sensitivity and specificity. When the meniscus is torn, the synovial fluid will enter the meniscus from the crack. As a result, the concentration of hydrogen protons in the internal meniscus increases, so the meniscus tear part will show high signal under MRI scan [20,21]. The MRI image varies according to the situation of tibial plateau fracture combined with meniscus injury and tear, and there are a variety of classification methods. As per the 4-level classification method of tibial plateau fracture combined with meniscus injury proposed by Kopf et al. (2020) [22], level 0 means that a normal meniscus 

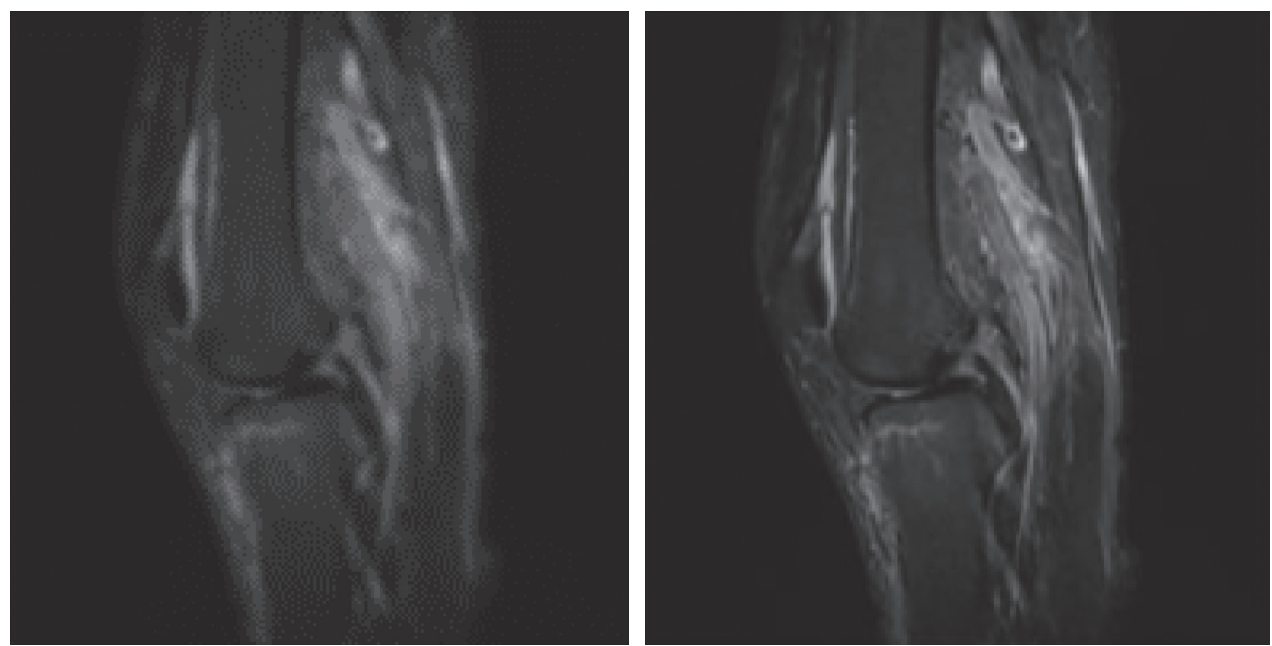

FIGURE 2: Reconstruction of MRI images of the knee joint based on deep learning (left is the original MRI image; right is the MRI image after reconstruction).
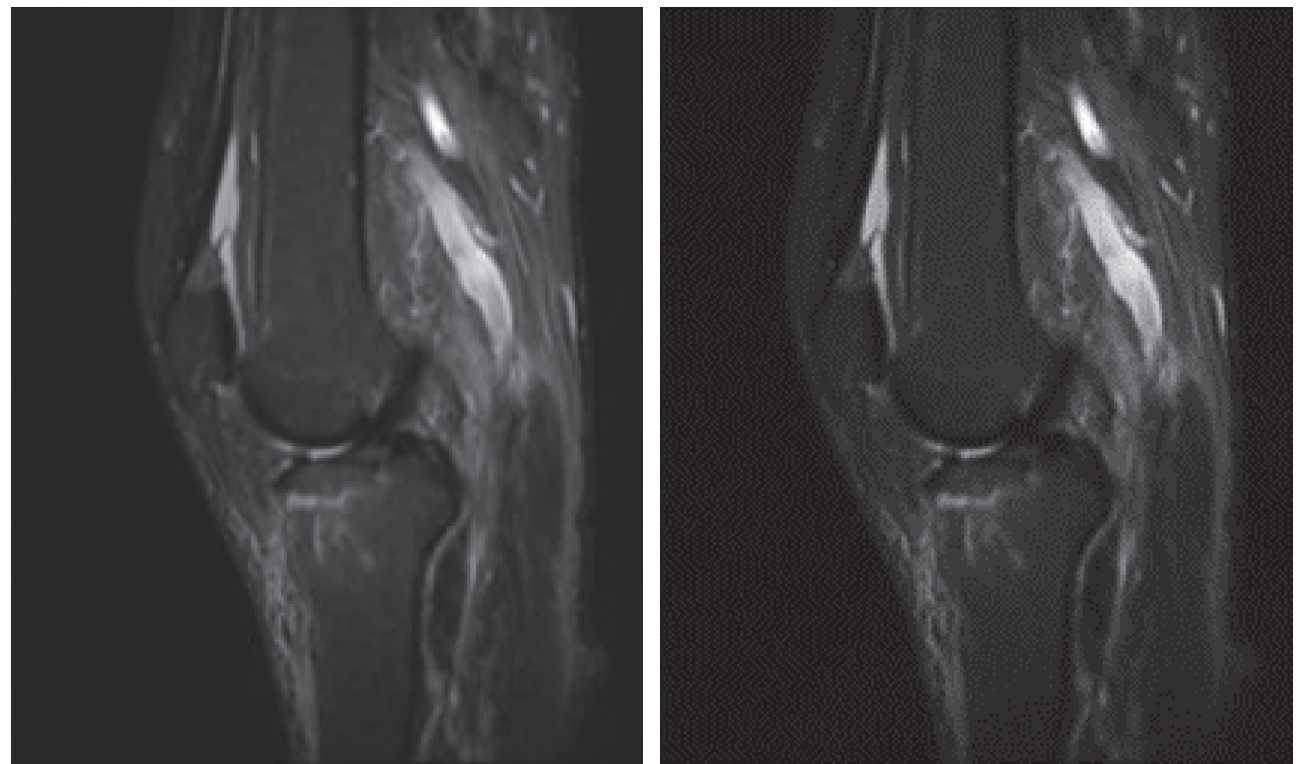

FIGURE 3: Restoration of blurred parts by CNN: (a) blurred image before restoration; (b) clearer image restored by the CNN algorithm.

shows a uniform low signal and the shape is intact; level I means that the meniscus appears as a shadow of enhanced signals; level II means that the meniscus shows high-intensity linear shadow that does not affect the articular surface; and level III means that the meniscus shows an abnormal high-intensity signal that affects the articular surface, and the appearance of three consecutive similar signals indicates that the meniscus is torn.

The MRI image data will appear blurry and has artifacts and noise due to limb movement, special examination parts, heartbeat, and breathing, so MRI images need to be reconstructed and optimized. The undersampling technique can effectively reduce the time spent in data collection process, but at the same time it will also affect the accuracy of the image. Deep learning provides a more convenient and fast method for improving the diagnostic efficiency in the medical field [23]. Hence, neural network models can be used to learn knee joint undersampling and fully sampling MRI image data for training. The trained model can optimize and reconstruct knee joint MRI images, not restricted by physical, physiological, and hardware conditions. According to the results, of the total 160 meniscuses in 80 patients with tibial plateau fracture combined with meniscus injury, 64 meniscuses were normal and 88 meniscuses were injured. The sensitivity, specificity, and accuracy of optimized MRI in diagnosing tibial plateau fracture with meniscus injury were $96.9 \%, 93.2 \%$, and $95.3 \%$, respectively. MRI images optimized by the deep learning reconstruction algorithm had high sensitivity for diagnosis of tibial plateau fracture combined with meniscus injury and had high 

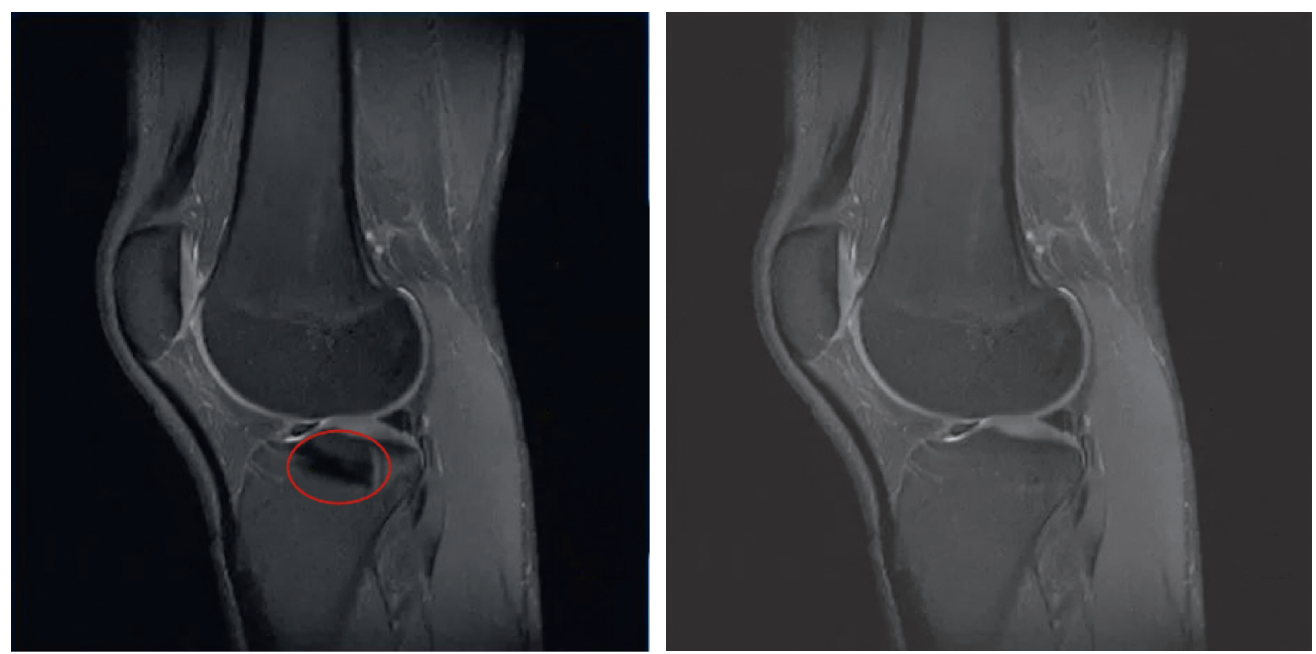

FIgURE 4: Restoration of the shadow part by CNN: (a) image with shadow before restoration; (b) deshadowed image restored by the CNN algorithm.

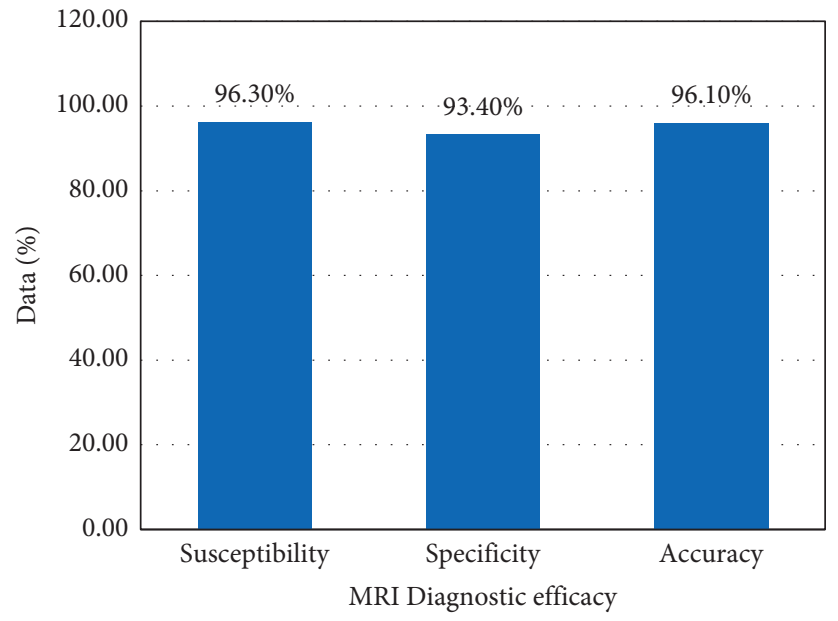

Figure 5: Diagnostic efficacy of MRI of tibial plateau fracture combined with meniscus injury.

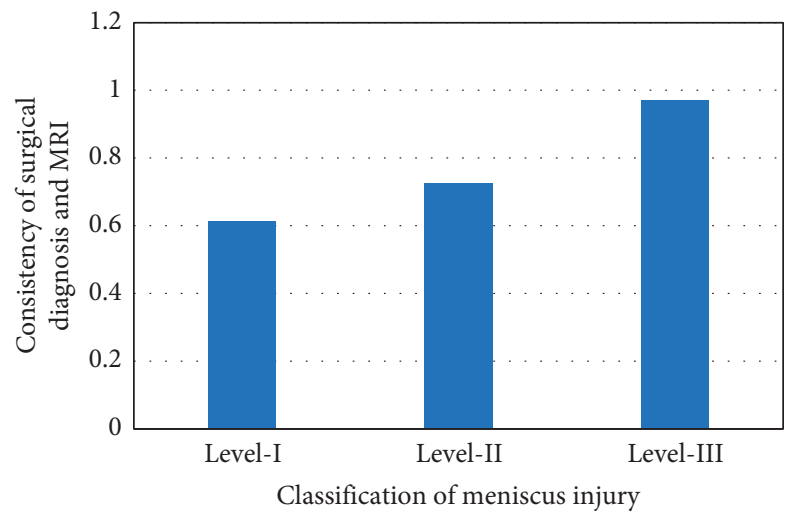

FIGURE 6: Consistency between intraoperative diagnosis and MRI diagnosis results. 


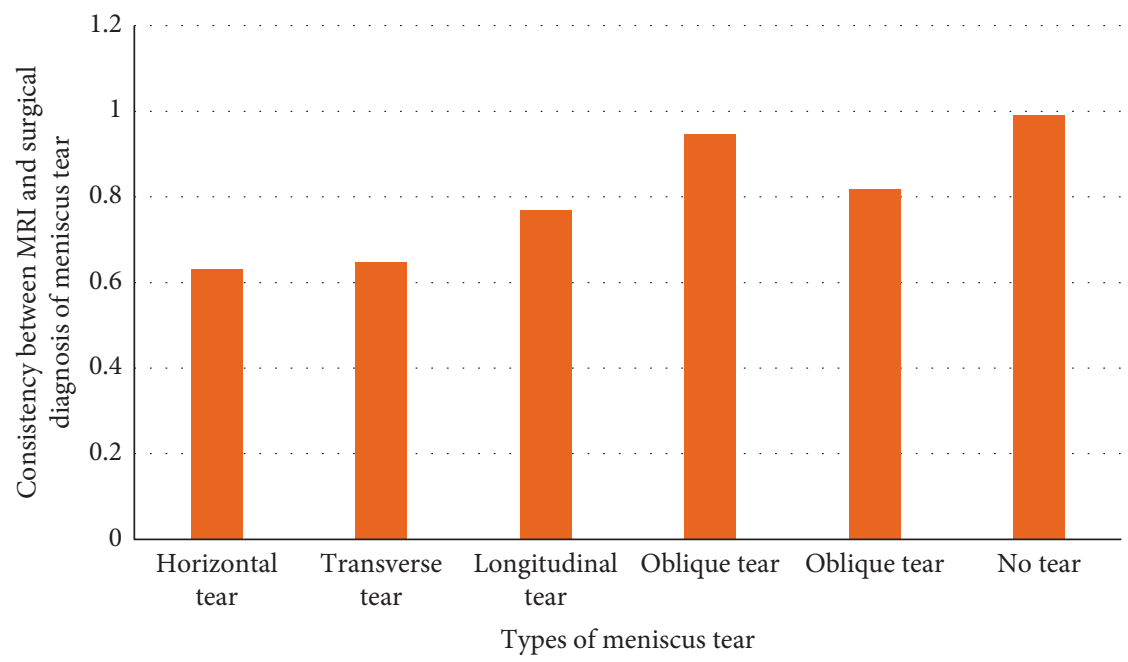

FIGURE 7: The consistency between MRI and intraoperative observation results of meniscus tear classification.

consistency with intraoperative results. This indicated that the optimized MRI image was effective in the diagnosis of tibial plateau fracture with meniscus injury.

\section{Conclusion}

In the study, the deep learning technology was used to reconstruct the MRI images of knee joint, to further improve the clinical diagnosis efficiency of tibial plateau fractures combined with meniscus injuries. The results showed that the reconstruction model based on the deep learning CNN algorithm can reconstruct and optimize MRI images of knee joint. The optimized MRI image had high sensitivity and accuracy in the diagnosis of tibial plateau fracture combined with meniscus injury and had a high consistency with the intraoperative results, suggesting that the optimized MRI image was effective in the diagnosis of tibial plateau fracture combined with meniscus injury. However, the shortcoming of this study is that the sample size included is small, so the one-sidedness and limitations of this study cannot be ignored. In the follow-up, an expanded sample size is necessary to strengthen the findings of the study. In conclusion, this study provides a basis and theoretical support for the subsequent treatment of knee joint fractures combined with meniscal injuries.

\section{Data Availability}

The data used to support the findings of this study are available from the corresponding author upon request.

\section{Conflicts of Interest}

The authors declare that there are no conflicts of interest.

\section{References}

[1] E. Vaienti, G. Scita, F. Ceccarelli, and F Pogliacomi, "Understanding the human knee and its relationship to total knee replacement," Acta Bio-Medica: Atenei Parmensis, vol. 88, no. 2S, pp. 6-16, 2017.
[2] W. Hönle, T. Schmickal, S. Lerzer, and A. Schuh, "Diagnostik am Kniegelenk [Clinical examination of the knee joint]," $M M W$ - Fortschritte der Medizin, vol. 160, no. 20, pp. 56-60, 2018.

[3] P. Graham, "Tlateau fracture," Orthopaedic Nursing, vol. 36, no. 4, pp. 303-305, 2017.

[4] P. Salari, G. Busel, and J. T. Watson, "A radiographic zonebased approach to predict meniscus injury in lateral tibial plateau fracture," Injury, vol. 52, no. 6, pp. 1539-1543, 2021.

[5] D. N. Greif, M. G. Baraga, M. G. Rizzo et al., "MRI appearance of the different meniscal ramp lesion types, with clinical and arthroscopic correlation," Skeletal Radiology, vol. 49, no. 5, pp. 677-689, 2020.

[6] S. Xie, Z. Yu, and Z. Lv, "Multi-disease prediction based on deep learning: a survey," Computer Modeling in Engineering and Sciences, vol. 128, no. 2, pp. 489-522, 2021.

[7] N. Phelan, P. Rowland, R. Galvin, and J. M. O’Byrne, “A systematic review and meta-analysis of the diagnostic accuracy of MRI for suspected ACL and meniscal tears of the knee," Knee Surgery, Sports Traumatology, Arthroscopy, vol. 24, no. 5, pp. 1525-1539, 2016.

[8] F. Lecouvet, T. Van Haver, S. Acid et al., "Magnetic resonance imaging (MRI) of the knee: identification of difficult-to-diagnose meniscal lesions," Diagnostic and Interventional Imaging, vol. 99, no. 2, pp. 55-64, 2018.

[9] K. Asai, J. Nakase, T. Oshima, K. Shimozaki, K. Toyooka, and $\mathrm{H}$. Tsuchiya, "Lateral meniscus posterior root tear in anterior cruciate ligament injury can be detected using MRI-specific signs in combination but not individually," Knee Surgery, Sports Traumatology, Arthroscopy, vol. 28, no. 10, pp. 30943100, 2020.

[10] M. Kfuri and J. Schatzker, "Revisiting the Schatzker classification of tibial plateau fractures," Injury, vol. 49, no. 12, pp. 2252-2263, 2018.

[11] A. A. Ozaslan, A. Alacaoglu, O. B. Demirel, T. Çukur, and E. U. Saritas, "Fully automated gridding reconstruction for non-Cartesian $\mathrm{x}$-space magnetic particle imaging," Physics in Medicine and Biology, vol. 64, no. 16, Article ID 165018, 2019.

[12] Y. Han, L. Sunwoo, and J. C. Ye, “ $\$\{k\} \$$-space deep learning for accelerated MRI," IEEE Transactions on Medical Imaging, vol. 39, no. 2, pp. 377-386, 2020. 
[13] C. Huang, "ReLU networks are universal approximators via piecewise linear or constant functions," Neural Computation, vol. 32, no. 11, pp. 2249-2278, 2020.

[14] L. Yao, "A multifeature extraction method using deep residual network for MR image denoising," Comput Math Methods Med, vol. 2020, Article ID 8823861, 2020.

[15] Z. Wan, Y. Dong, Z. Yu, H. Lv, and Z. Lv, "Semi-supervised support vector machine for digital twins based brain image fusion," Frontiers in Neuroscience, vol. 15, Article ID 705323, 2021.

[16] A. J. S. Fox, F. Wanivenhaus, A. J. Burge, R. F. Warren, and S. A. Rodeo, "The human meniscus: a review of anatomy, function, injury, and advances in treatment," Clinical Anatomy, vol. 28, no. 2, pp. 269-287, 2015.

[17] M. Hu, Y. Zhong, S. Xie, H. Lv, and Z. Lv, "Fuzzy system based medical image processing for brain disease prediction," Frontiers in Neuroscience, vol. 15, Article ID 714318, 2021.

[18] L. Ghazi Zadeh, A. Chevrier, J. Farr, A R. Scott, and D. B. Michael, "Augmentation techniques for meniscus repair," Journal of Knee Surgery, vol. 31, no. 1, pp. 99-116, 2018.

[19] M. Jarraya, F. W. Roemer, M. Englund et al., "Meniscus morphology: does tear type matter? A narrative review with focus on relevance for osteoarthritis research," Seminars in Arthritis and Rheumatism, vol. 46, no. 5, pp. 552-561, 2017.

[20] Y. Okazaki, T. Furumatsu, S. Masuda et al., "Pullout repair of the medial meniscus posterior root tear reduces proton density-weighted imaging signal intensity of the medial meniscus," Acta Medica Okayama, vol. 72, no. 5, pp. 493-498, 2018.

[21] R. Chapin, "Imaging of the postoperative meniscus," $R a$ diologic Clinics of North America, vol. 56, no. 6, pp. 953-964, 2018.

[22] S. Kopf, P. Beaufils, M. T. Hirschmann et al., "Management of traumatic meniscus tears: the 2019 ESSKA meniscus consensus," Knee Surgery, Sports Traumatology, Arthroscopy, vol. 28, no. 4, pp. 1177-1194, 2020.

[23] S. Guo, R. Chen, H. Li, T. Zhang, and Y. Liu, "Identify severity bug report with distribution imbalance by CR-SMOTE and ELM," International Journal of Software Engineering and Knowledge Engineering, vol. 29, no. 2, pp. 136-175, 2019. 